\title{
Maximal sum-free sets in abelian groups of order divisible by three
}

\section{Anne Penfold Street}

A subset $S$ of an additive group $G$ is called a maximal sum-free set in $G$ if $(S+S) \cap S=\emptyset$ and $|S| \geq|T|$ for every sum-free set $T$ in $G$. In this note, we prove a conjecture of Yap concerning the structure of maximal sum-free sets in finite abelian groups of order divisible by 3 but not divisible by any prime congruent to 2 modulo 3 .

Given a finite additive abelian group $G$ and non-empty subsets $S, T$ of $G$, let $S+T$ denote the set $\{s+t \mid \varepsilon \in S, t \in T\}, \bar{S}$ the complement of $S$ in $G$ and $|S|$ the cardinality of $S$. Define the subgroup $H(S)$ by $H(S)=\{g \in G \mid S+g=S\}$ so that

(a) $S+H(S)=S$ and

(b) if $S+K=S$ for some subgroup $K$, then $K \leq H(S)$.

Note that the subgroup generated by $H(S)$ and $H(T)$ is contained in $H(S+T)$. We call $S$ a sum-free set in $G$ if $(S+S) \subseteq \bar{S}$. If, in addition, $|S| \geq|T|$ for every sum-free set $T$ in $G$, then we call $S$ a maximal sum-free set in $G$.

Suppose that $|G|$ is not divisible by any prime congruent to 2 modulo 3 , but is divisible by 3 . Diananda and Yap [1] showed that if $S$ is a maximal sum-free set in $G$, then

(a) $|S|=|G| / 3$ and

(b) $S$ is a union of cosets of a subgroup $H$ of index $3 n$ in $G$

Received 24 January 1972. 
(for some $m$ ) such that either

$$
\begin{aligned}
& \text { (i) }|S+S|=2|S|-|H| \text { or } \\
& \text { (ii) }|S+S|=2|S| \text { and } S \cup(S+S)=G \text {. }
\end{aligned}
$$

Yap [3] conjectured that case (ii) cannot in fact occur. Here we prove his conjecture. Part of the proof in [1] is restated for convenience.

Let $A$ be a subset of $G$ such that $A=-A$. Since $|G|$ is odd, $|A|$ is odd if and only if $0 \in A$.

$0 \mid S$ and $|S|$ is odd, so $S \neq-S$. $0 \in S-S=-(S-S)$, so $|S-S|$ is odd. Since $S$ is sum-free, $S \cap(S+S)=\emptyset=(S \cup(-S)) \cap(S-S)$.

We apply Kneser's Theorem: the statement given in [2] is the most convenient. If $H=H(S+S)$, then $S+S+H=S+S$ and either $|S+S| \geq 2|S|$ or $|S+S|=2|S+H|-|H|$.

If $S+H$ is not sum-free, then for some $s \in S, h \in H$ we have $8+h \in(S+H)+(S+H)=S+S+H=S+S$. But then

$\boldsymbol{s} \in S+S-h=S+S$ and $S$ is not sum-free, which is a contradiction. Hence $S+H$ is a sum-free set containing $S$. By the maximality of $S$, $S+H=S$ and hence $H(S)=H$.

Since $S$ is sum-free, $|S+S| \leq 2|S|$ and the possibilities for $|S+S|$ become

(i) $|S+S|=2|S|-|H|$ or

(ii) $|S+S|=2|S|$.

We know that $S-S+H=S-S$, so $H \leq H(S-S)$. A proof similar to that for $S+H$ shows that $S+H(S-S)=S$ also. Hence $H(S-S)=H$.

Then the possibilities for $S-S$, just as for $S+S$, are

(i) $|S-S|=2|S|-|H|$ or

(ii) $|S-S|=2|S|$.

But $|S-S|$ is odd, so (ii) is ruled out and $|S-S|=2|S|-|H|$.

If $|S+S|=2|S|$, then exactly one coset of $H$ occurs in $S+S$ but not in $S-S$. We show this is impossible.

Since $S$ is a union of cosets of $H$ and $S \neq-S$, we have $|S \cup(-S)| \geq|S|+|H|$. Since $(S \cup(-S)) \cap(S-S)=\emptyset$, we have 
$|S \cup(-S)| \leq|S|+|H|$. Hence $|S \cup(-S)|=|S|+|H|,|S n(-S)|=|S|-|H|$ and $S$ consists of a union of pairs of cosets, $g_{i}+B$ and $-g_{i}+A$, $i=1, \ldots, n$ for some $n$, together with one coset $g+H$ whose negative is not contained in $S$.

We want a representative of the coset of $H$ which is contained in $s+s$ but not in $s-s$. Now $g_{i}+g_{j}=g_{i}-\left(-g_{j}\right)$ and $g+g_{j}=g-\left(-g_{j}\right)$, so both belong to $s-s$. So the only possibility is $2 g$. Since $|G|$ is odd, $2 g \neq-2 g$ and we consider the coset $-2 g+H \subseteq S+S$. If $-2 g=g+g_{j}$, then $2 g=\left(-g_{j}\right)-g \in S-S$; if $-2 g=g_{i}+g_{j}$, then $2 g=\left(-g_{i}\right)-\left(g_{j}\right) \in S-S$.

In either case we have a contradiction, so $|S+S| \neq 2|S|$.

\section{References}

[1] Palahenedi Hewage Diananda and Hian Poh Yap, "Maximal sum-free sets of elements of finite groups", Proc. Japan Acad. 45 (1969), 1-5.

[2] J.H.B. Kemperman, "On small sumsets in an abelian group", Acta Math. $103(1960), 63-88$.

[3] Hian-Poh Yap, "Structure of maximal sum-free sets in groups of order 3p ", Proc. Japan Acad. 46 (1970), 758-762.

Department of Mathematics,

University of Queensland,

St Lucia,

Queensland. 